\title{
Relevant communication skills for pregnant women - a dental perspective
}

\begin{abstract}
Regular dental care throughout pregnancy is essential for the mother and the fetus. Education regarding the importance of oral health during pregnancy as well as after child birth can prevent potential dental problems in the pregnant and the pediatric population. Awareness and knowledge about the maintenance of oral health during and after pregnancy should be dissipated by the dentist in a way which is easy for patient to understand and decipher and also comply with. Therefore dentist should utilize appropriate communication skills for educating the pregnant women to deem oral health maintenance as important as any other part of body.
\end{abstract}

Keywords: pregnancy, communication skills, dental, pregnant women, oral health, challenges, pregnancy, cardiogenic bacteria, maternal attitudes
Volume 2 Issue 3 - 2017

\author{
Varsha Murthy,' Sethuraman KR, ${ }^{2}$ Sunayana \\ Choudhury, ${ }^{3}$ Shakila $\mathrm{R}^{4}$ \\ 'Department of Prosthodontics and Implantology, Mahatma \\ Gandhi Medical College Campus, India \\ ${ }^{2}$ Department of General Medicine, Sri Balaji Vidyapeeth institute, \\ India \\ ${ }^{3}$ Department of Clinical Psychology and Psychiatry, Mahatma \\ Gandhi Medical College and Research institute, India \\ ${ }^{4}$ Department of Prosthodontics and Crown \& Bridge, Mahatma \\ Gandhi Postgraduate Institute of Dental Sciences, India
}

\begin{abstract}
Correspondence: Varsha Murthy, MDS (Prosthodontics), Professor, Department of Prosthodontics and Implantology, Indira Gandhi Institute of Dental Sciences. Mahatma Gandhi Medical College Campus, Sri Balaji Vidyapeeth, Pondy-Cuddalore Main Road, Pillayarkuppam, Puducherry 607402, India, Tel +9I 7708364959,Email drvarshamurthy@gmail.com
\end{abstract}

Received: April 21, 2017| Published: May II, 2017

\section{Introduction}

Lack of awareness among pregnant women, results in pregnancy complications and pediatric dental problems which can be prevented if diagnosed early. Appropriate dental care during pregnancy can improve maternal oral health, reduce mother to fetus transmission of cardiogenic bacteria, and create opportunities for preventive supervision. ${ }^{1}$ Poor maternal oral health increases the risk of pregnancy complications including preterm delivery, low birth weight, gestational diabetes, preeclampsia, stillbirth etc. Oral flora and oral health of mother are one of the greatest predictors of childhood oral flora and oral health. ${ }^{2}$ Fetal exposure to oral pathogens may increase risk of subsequent neonatal intensive care admission. ${ }^{3}$ Maternal attitudes and behaviors towards oral hygiene and dietary practice also may influence this risk. ${ }^{4}$

Studies have reported that barriers due to dentist and patients factors contribute to low rates of service utilization. ${ }^{2}$ According to dentist's, high levels of perceived time and economic costs, dissatisfaction with compensation by insurers, staff resistance, skill/training costs and peer pressure from physicians are significant barriers to provision of care for pregnant patients. ${ }^{3}$ Dentists' attitudes also have significant negative impacts as dentists with incorrect knowledge may perceive substantial barriers, thus avoiding care of pregnant patients. ${ }^{3}$

Most of the women do not consider it important to have a dental check up during pregnancy. ${ }^{5}$ Majority of the women have a poor understanding of the relationship between maternal oral health and infant oral health, knowledge of infant oral health, awareness regarding the timing of the first dental visit, benefits of fluoride etc. ${ }^{5}$ The possible barriers to utilization of dental services include pregnancy stressors(domestic relationships, finances, and employment), perception of dental experience, attitude toward dentist, importance and valuing of oral health, perceived ability to pay for care, time constraints, and attitude of dental practitioners' and staff towards them. ${ }^{1,6}$

Patients perceptions and erroneous beliefs about the safety of dental treatment (for example-dental treatment can harm the fetus, believing that poor oral health is normal and accepted during pregnancy) may contribute to the low rate of service utilization which needs to be corrected through oral health education of pregnant women, oral care and prenatal care providers. ${ }^{7,8}$

Pregnant women who visit dentist were more likely to be those who received oral health education during the prenatal period and are aware about the potential consequences of poor maternal oral health. ${ }^{7}$ Oral health education play an important role in enhancing knowledge and awareness for mothers' to seek oral health care behavior. ${ }^{7,9}$ There is a need for specialized training for health providers specially obstetricians, dentist and pediatricians on communication strategies that promote oral health for pregnant women in accessible and timely manners. $^{2}$

There is growing evidence that good oral health of the pregnant female right from the pregnancy could be the key in establishing good infant oral health. ${ }^{5}$ Therefore, women's health issues should be given a higher priority by policy decision makers, health care providers, and public and private insurers as it significantly influences the future of oral and general health of the next generation. ${ }^{3}$

\section{Discussion}

\section{Challenges in management of pregnant dental patien- ts}

There are some challenges during interaction with pregnant women for dental treatment. Some of them are: 
a. Physiological changes taking place in a woman make them more vulnerable and dependent on people around them for opting or undergoing any treatment.

b. Such changes make them more tired and sick thus compromising the cooperation for any lengthy treatment procedure.

c. Many women have myths and misconceptions about oral health during pregnancy causing avoidance from seeking oral health care.

d. Women sometimes may not disclose to the dentist regarding the pregnancy as they may feel that it is of no relevance to the dentist.

e. Females belonging to villages, orthodox families and low socio economic status have a myth that disclosing pregnancy before three months to anyone might harm the baby.

f. Orthodox females feel shy to tell by themselves and it's only when dentist starts to do some procedures that the patient comes out of the fact that they are pregnant.

g. Dental procedures usually are not recommended during the first trimester owing to the risk posed by teratogens during organogenesis and risk of spontaneous abortions which could be attributed to the dental treatment. ${ }^{2}$

h. The females usually experience morning sickness in early part of pregnancy and cannot cooperate for the procedures.

i. Increased sensitivity of smell and taste in pregnancy may lead to dislike of them and can cause symptoms of gagging, nausea, and vomiting. Therefore, such dental materials should be avoided. ${ }^{2}$

j. For a majority of pregnant women across all socioeconomic levels substantial gaps in the overall oral health literacy, knowledge, misunderstandings, and a lack of dental care access exists. ${ }^{5,9}$

k. Significant gaps exist in women's knowledge about the risk for certain oral diseases in pregnancy which make them to ignore the negative changes in their oral health, such as bleeding gums, as a normal and accepted part of pregnancy that do not necessitate treatment. ${ }^{2,10}$

1. Substantial gaps also exist in women's knowledge about the oral care of infants. Studies have found that they did not know that it is important to wipe infants' gums after they drink from a breast or bottle. ${ }^{4}$ It has also been found that most women reported they would start cleaning their child's oral cavity only once the first tooth erupts and most of them visit the dentist only if the child has a problem. ${ }^{5}$

\section{Methods to encounter challenges using relevant com- munication skills-}

In dealing with pregnant women following attributes of effective communication are relevant-the information provided by the health care provider should be accurate, available at right time to the user, understandable, culturally competent, from a reliable source, evidence based, should be balance between benefit and risk, consistent over time and should be repeated to reinforce..$^{11,12}$ Following skills can be applied for any given situation while dealing with pregnant women as well the family members ${ }^{11,12}$

Understanding and respecting patient's perspectives, ideas, and beliefs: To assess the existing ideas, beliefs and practices of the patient and clarify patient's myths and misconceptions (based on their ethnicity and religious beliefs) regarding the oral health using appropriate evidence based examples.

\section{Questioning and listening skills}

a. To elicit the existing knowledge of the patient regarding the dental treatment.

b. To assess the family support (husband or any relatives who could help her in decision making and coming for the appointments).

c. To assess the financial status whether the patient will be able to carry on treatment till its completion.

d. To elicit past dental history (satisfactory/unsatisfactory) by using questioning skills.

e. If patient gives unsatisfactory past dental history then explore more using open or closed ended/direct or indirect/leading or non leading questions as to whether the dissatisfaction was with the dentist or the treatment.

f. To elicit any underlying fear or phobia regarding the treatment.

g. To find if the patient has any doubts to be clarified from the dentist.

h. If patient still shows resistance to reveal any information then accompanying family members can be enquired.

\section{Answering skills}

a. Answering to their queries till they are satisfied helps in getting more adherence of treatment from the patient.

b. While answering the queries, educate them using evidence based literature and aids (pamphlets, educational videos).

c. Have control over the dyad. Empathize, reassure and acknowledge the patient whenever required. ${ }^{11,12}$

\section{Rapport building skills}

a. Rapport building will help the dentist in building trust with the patient.

b. Patients should be explained using words that are simple, clear and easy to understand with lot of examples. Avoid using jargons and complicated technical language.

c. Elicit patient agenda.

d. Build therapeutic relationship with the patient.

\section{Non verbal behavior}

a. Dentist should be able to observe and understand the non verbal behavior (e.g.-open versus closed position, positive versus negative signals, signals of thinking, skepticism, disinterest and restlessness etc $)^{11,12}$ of the patient as well as the family members who accompany the patient, regarding the treatment plan.

b. Signals of pain, discomfort, anxiety, fear, dissatisfaction with the dentist's opinion should be elicited and addressed.

c. Fussy and pampered females in early pregnancy are to be handled with caution.

\section{Counseling and motivation skills}

a. To educate the patient and family regarding maintenance of maternal oral hygiene and nutrition of pregnant female. 
b. To educate the patient and family regarding the harmful effects of radiographs on the fetus and thus avoidance of frequent radiographs unless an emergency and to postpone the dental treatments until second trimester unless it is an emergency.

c. To educate the patient regarding oral changes that takes place during the course of pregnancy (pregnancy cyst, gingivitis, periodontitis, gingival hyperplasia, pyogenic granuloma etc).

d. To educate and motivate the patient regarding the importance of dental treatment in pregnancy and how it could have influence on the new born.

e. To educate patients regarding the oral hygiene care of infants and the importance of regular dental checkup of infants.

f. Patient's adherence and compliance after the procedures for success of treatment.

\section{Motivational interviewing}

a. Motivational interviewing has been used to promote the use of dental care for the parents of preschoolers and also with pregnant women..$^{2,3,13}$

b. This method of instruction provides the least intimidating and most responsive form of interaction between a dentist and the patient. ${ }^{13}$

\section{Persuasion skills}

a. To insist on the importance of regular check up during and after pregnancy for the mother.

b. In making they understand the importance of regular dental checkups for infants and children during growing years (diagnosis of feeding bottle caries, anodontia, delay in eruption of teeth, orthodontic misalignments' etc).

\section{Communicating prognosis and risks}

a. Avoid doing any treatment without consulting and communicating with patient's obstetrician/physician.

b. Explain the patient completely about the treatment.

c. Obtain written informed consent.

d. Avoid treatments in early and later part of pregnancy.

e. Help patient in decision making especially complex decisions.

\section{Negotiating skills}

a. Make the appointments flexible (short and convenient) for patients to get adherence.

b. Explain them the anticipated problems and explain what to do in such situations. Give a contact number or address in case of emergency.

c. In case if something goes wrong, take responsibility and arrange for management.

Preliminary assessment of patient psychology: Dentist should be aware of basic assessment of patient psychology to elicit patient's perception regarding the dental treatment.

\section{Conclusion}

Good oral health during pregnancy can not only improve the quality of life of the pregnant mother, but also potentially reduce complications during pregnancy as well as the risk of the child developing oral health problems in the future. Therefore, it is important to understand pregnant women's oral health knowledge and behaviors and to identify barriers to accessing care and practicing good oral hygiene. Further, improved efforts are needed to communicate oral health information to women before, during, and after pregnancy. To be effective, communication efforts must reach pregnant women at the appropriate time and must be communicated to them in a form that they easily understand.

\section{Acknowledgements}

None.

\section{Conflict of interest}

Author declares that there is no conflict of interest.

\section{References}

1. Le, M, Riedy C, Weinstein P, et al. Barriers to utilization of dental services during pregnancy: a qualitative analysis. J Dent Child (Chic). 2009;76(1):46-52.

2. Vt H, TM, TS, et al. Dental considerations in pregnancy-a critical review on the oral care. J Clin Diagn Res. 2013;7(5):948-953.

3. Lee RS, Milgrom P, Huebner CE, et al. Dentists' Perceptions of Barriers to Providing Dental Care to Pregnant Women. Womens Health Issues. 2010;20(5):359-365

4. Boggess KA, Urlaub DM, Moos MK, et al. Knowledge and beliefs regarding oral health among pregnant women. J Am Dent Assoc. 2011;142(11):1275-1282.

5. Gambhir RS, Nirola A, Gupta T, et al. Oral health knowledge and awareness among pregnant women in India: A systematic review. J Indian Soc Periodontol. 2013;19(6):612-617.

6. Detman LA, Cottrell BH, Denis Luque MF. Exploring dental care misconceptions and barriers in pregnancy. Birth. 2010;37(4):318-324.

7. Saddki N, Yusoff A, Hwang YL. Factors associated with dental visit and barriers to utilisation of oral health care services in a sample of antenatal mothers in Hospital Universiti Sains Malaysia. BMC Public Health. 2010;10:75.

8. George A, Johnson M, Duff M, et al. Midwives and oral health care during pregnancy: perceptions of pregnant women in south-western Sydney, Australia. J Clin Nurs. 2011;21(7-8):1087-1096.

9. Habashneh R, Guthmiller JM, Levy S, et al. Factors related to utilization of dental services during pregnancy. J Clin Periodontol. 2005;32(7):815-821.

10. Keirse M, Plutzer K. Women's attitudes to and perceptions of oral health and dental care during pregnancy. J Perinat Med. 2010;38(1):3-8.

11. Sethuraman KR. Communication skills in clinical practice (Doctor patient communication). 1st ed. India: Jaypee Medical Publishers P Ltd; 2001.

12. Sethuraman KR. Communication skills in clinical practice (Doctor patient communication). 2nd ed. India: Jaypee Medical Publishers P Ltd; 2017.

13. Andrew Zima. The effects of motivational interviewing in pregnancy on knowledge and retention of infant oral health information. USA: Thesis. Virginia Commonwealth University; 2010. 